\title{
Risiko Gestagene: Vor allem junge Frauen werden unter der Pille depressiv
}

\author{
Manche Frauen fallen mit Kontrazeptiva in ein \\ Stimmungstief. Vor allem solche unter 20 Jahren mit \\ reinen Gestagenpräparaten sind gefährdet - bei \\ innen ist das Depressionsrisiko zum Teil dreifach \\ erhöht.
}

Es kommt immer wieder vor: Ein Frau fällt in eine schwere Depression, kurz nachdem sie begonnen hat, die Antibabypille zu nehmen. Vor allem Präparate mit hohem Gestagenanteil scheinen das Risiko für eine Depression zu erhöhen, erläuterte Professor Volker Arolt von der Universität Münster. Allerdings waren Studien zu diesem Thema bislang recht widersprüchlich, manche konnten ein Zusammenhang erkennen, andere jedoch nicht, sagte Arolt auf der Fortbildungsveranstaltung „Psychiatrie Update" in Mainz. Möglicherweise lag dies an den unterschiedlichen Präparaten, die in solchen Studien analysiert wurden, und am Alter: Nach Daten einer großen prospektiven Registeranalyse aus Dänemark sind primär junge Frauen unter reinen Gestagenpräparaten gefährdet, mit dem Alter nimmt das hormonell bedingte Depressionsrisiko wieder ab.

\section{Depressionsgefahr vor allem im ersten Anwendungsjahr erhöht}

Für die Studie wurden Angaben aus den Jahren 2000 bis 2014 $\mathrm{zu}$ allen dänischen Frauen im Alter von 15 bis 34 ausgewertet, die noch keine Antidepressiva genommen und auch noch keine Depressionsdiagnose hatten. Die Forscher interessierten sich dabei sowohl für den Gebrauch von Antikonzeptiva als auch den von Antidepressiva in den folgenden Jahren. $55 \%$ der über eine Million Frauen setzten auf hormonelle Verhütungsmittel, und 133.000 nahmen im Untersuchungszeitraum von im Mittel 6,4 Jahren erstmals Antidepressiva.

Im Vergleich zu Frauen ohne hormonelle Verhütungsmittel benötigten solche mit oralen Kontrazeptiva etwa 20 \% häufiger erstmals Antidepressiva, etwa $30 \%$ höher war diese Rate bei Frauen mit reinen Gestagenpräparaten. „Das klingt jetzt nicht nach großen Unterschieden“, so Arolt. Diese wurden jedoch deutlich, als die dänischen Forscher auf das Alter der Frauen schauten. Junge Frauen zwischen 15 und 19 Jahren benötigten unter reinen Gestagenpräparaten 2,2-fach häufiger Antidepressiva, unter nicht oralen hormonellen Kontrazeptiva wie Vaginalring und Hormonspirale mit Levonorgestrel sogar dreifach häufiger als Gleichaltrige ohne hormonelle Verhütung ( $\triangleright$ Abb. 1). „Dies liegt wohl daran, dass bei solchen Systemen besonders viele Gestagene in die Blutbahn gelangen“, so Arolt. Ähnlich stark erhöht war bei den jüngsten Frauen die Rate für eine erste Depressionsdiagnose. Bei Älteren ließ sich dagegen unter Kontrazeptiva kaum noch eine erhöhte Depressionsinzidenz nachweisen.

Das Risiko für eine depressive Verstimmung ist nach diesen Resultaten vor allem im ersten Jahr erhöht, danach nimmt es mit der Zeit ab. Arolt zieht daraus den Schluss: „Vorsicht mit progesteronhaltigen Antikonzeptiva bei Frauen unter 25 Jahren!“ Diese sollten seiner Ansicht nach zumindest keine reinen Gestagenpräparate sein.

Die Ursachen für die Stimmungseintrübung unter Gestagenen sind noch weitgehend unklar. Bekannt ist lediglich, dass für die Stimmung relevante Hirnregionen wie Amygdala, vorderes Cingulum und Gyrus frontalis inferior zyklusabhängig auf Umweltreize reagieren. Vermutet wird, so der Psychiater, dass Progesteron dabei GABA-Rezeptoren beeinflusst und den MAO-ASpiegel erhöht, was wiederum verminderte Serotoninspiegel zur Folge hat.

Thomas Müller, Springer Medizin

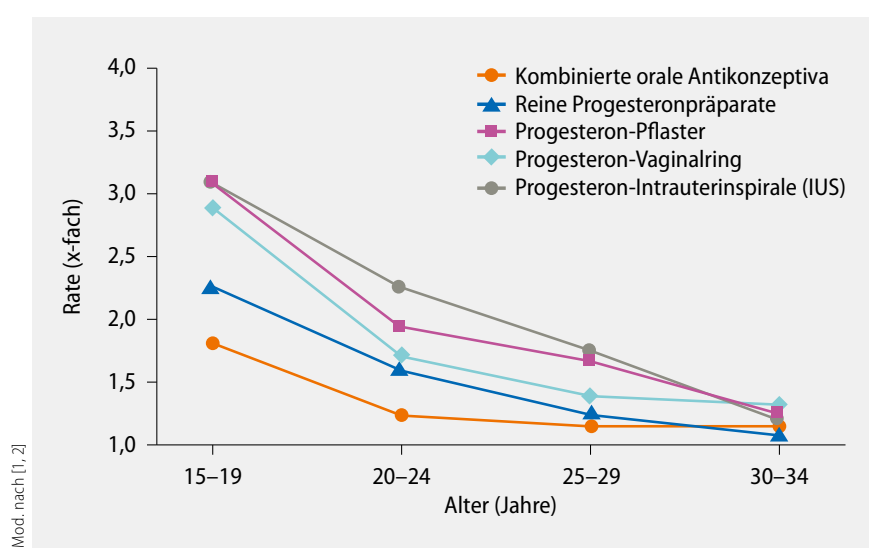

1 Bei jungen Frauen ist das Depressionsrisiko unter nicht oralen Kontrazeptiva mit Levonorgestrel wie Vaginalring und Hormonspirale am höchsten $(1,0=$ ohne Verhütungsmittel).

\begin{abstract}
Depression (Volker Arolt). Psychiatrie Update, Mainz, 24.3.2017
Literatur

Skovlund CW et al. JAMA Psychiatry 2016; 73 (11): 1154-62

2. Toffoletto et al. Psychoneuroendocrinology 2014; 50: $28-52$
\end{abstract}

